\title{
Thickness Dependence of Orientation Factors of Crystal Axes in Poly(vinylidene fluoride/ trifluoroethylene) Single Crystalline Films
}

\author{
Mohammad A. BARIQUE, Masanori SATO, ${ }^{\dagger}$ and Hiroji OHIGASHI ${ }^{\dagger+}$ \\ Department of Polymer Science and Engineering, Faculty of Engineering, \\ Yamagata University, Yonezawa 992-8510, Japan
}

(Received June 30, 2000; Accepted October 18, 2000)

\begin{abstract}
Poly(vinylidene fluoride/trifluoroethylene)single crystalline (SC) films, which were prepared by uniaxial stretching followed by crystallization in the paraelectric phase, are highly double-orientated, that is, the orthorhombic $c$-axis, and the axes perpendicular to the (110)/(200) planes of fully extended chain crystals, are preferentially oriented parallel to the stretching axis and normal to the film surface, respectively. The thickness dependence of orientation distributions of these axes in SC films were evaluated using wide-angle X-Ray diffraction. The results revealed that the orientation factors of the $c$-axis and the axes perpendicular to the $(110) /(200)$ planes increase with decreasing film thickness. It is suggested that the preferential orientation of the axes perpendicular to the (110)/(200) planes to the direction parallel to the film normal, which is developed in the hexagonal phase, is very high near the film surface, but it becomes gradually weaker as the distance from the surface increases. The formation mechanism of the orientational order is discussed, and a model for the preferential orientation distribution of the crystal planes is proposed.

KEY WORDS P(vinylidene fluoride/trifluoroethylene) Copolymer / Single Crystalline Film / Double Orientation / Orientation Distribution / Crystallization /
\end{abstract}

Much attention has been paid so far to the copolymers of vinylidene fluoride and trifluoroethylene, $\mathrm{P}(\mathrm{VDF} /$ TrFE), due to their prominent ferroelectricity, strong piezoelectricity and widespread applications to various piezoelectric devices, especially to ultrasonic transducers. These properties are highly dependent on the orientation of the crystal axes, and hence the properties can often be tailored by controlling the orientation of the polymer chains. Therefore it is of considerably practical and scientific interest to understand the orientation mechanism of $\mathrm{P}(\mathrm{VDF} / \mathrm{TrFE})$ copolymer films.

Ohigashi et al. ${ }^{1}$ first found that a single crystalline (SC) film of $\mathrm{P}(\mathrm{VDF} / \mathrm{TrFE})$ is formed by crystallization of a uniaxially stretched film in the hexagonal (paraelectric) phase under the condition that both surfaces of the film are made free from inhomogeneous stresses arising from contact with solid materials. In this highly crystallized film, the orthorhombic [001] axis and the axes perpendicular to the (110) and/or (200) planes of fully extended chain crystals are preferentially oriented parallel to the stretching axis and normal to the film surface, respectively. Owing to such unique structure, the SC films have mechanical and piezoelectric properties much superior to those of lamellar crystalline films. ${ }^{1,2}$

The preferential orientation of chain molecules in fluoro-polymers is very sensitive to the boundary condition at the film surface as in $\mathrm{P}(\mathrm{VDF} / \mathrm{TrFE})$. Wittmann and $\mathrm{Smith}^{3}$ investigated friction-transfer thin layers of a single crystal-like film of polytetrafluoroethylene (PTFE). The observed chain orientation and crystal perfection were very high. They found that the PTFE thin layer has ability to induce oriented growth of several organic compounds and polymers deposited on the thin layer. Moncton et $a l .^{4}$ worked on freely suspended smectic liquid crystal films from 2 to 100 layers thick and they found well-defined lattice orientational order in the thin films.

Many studies have been performed on the chain conformations, crystal structures, phase transition, electrical and mechanical properties of $\mathrm{P}(\mathrm{VDF} / \mathrm{TrFE})$, but to our knowledge the orientation mechanism of crystallites in $\mathrm{P}(\mathrm{VDF} / \mathrm{TrFE})$ copolymer films has not been reported so far. In the present paper, we report the thickness dependence of orientation factors of crystal axes and the orientation mechanism of $\mathrm{P}(\mathrm{VDF} / \mathrm{TrFE})$ single crystalline films.

Since this paper deals with the orientaion of the crystal axes or crystal planes of crystallites both in the orthorhomic (ferroelectric) phase and hexagonal (paraelectric) phase, we often need to use the directions perpendicular to the crystallographic planes. Then, we use in this paper the expression " $h k l$ axis" for the axis or direction perpendicular to the crystallographic plane $(h k l)$. The $h k l$ axis is parallel to the reciprocal lattice vector $h k l$.

\section{EXPERIMENTAL}

The sample used in this study was a $75 / 25 \mathrm{~mol} \%$ vinylidene fluoride and trifluoroethylene copolymer, which was kindly provided by Daikin Kogyo Co. Ltd. $\mathrm{P}(\mathrm{VDF} / \mathrm{TrFE})$ films were prepared by casting from an $N$, $N$-dimethylformamide solution onto glass plates at room temperature. Prior to the film casting, the solution was filtered through a glass filter in order to remove dust particles. The cast films were dried in an oven at $90^{\circ} \mathrm{C}$ for $2 \mathrm{~h}$ to ensure complete solvent removal. The uniaxially oriented films with various thickness were prepared by stretching the cast films to around five times the original length at room temperature. The stretched films

\footnotetext{
${ }^{\dagger}$ Present address: Anelva Co., 5-8-1 Yotsuya, Fuchu, Tokyo 183-8508, Japan.

${ }^{\dagger \dagger}$ To whom correspondence should be addressed.
} 


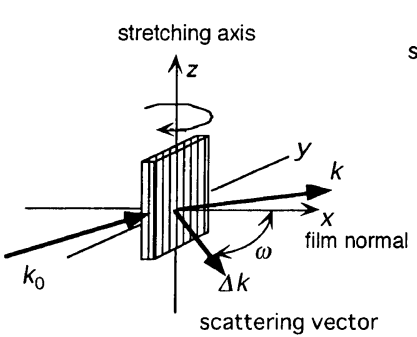

(a)

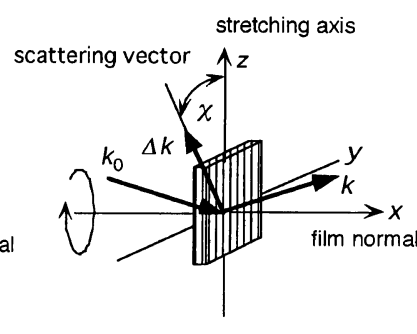

(b)
Figure 1. Sample setting configuration for measurements of orientation distribution of crystal axes in SC films by X-Ray diffraction: (a) the X-Ray scattering vector $\Delta k=k-k_{0}$ is scanned in the $x y$ plane for measurement of distribution of the $110 / 200$ axes $\left(k_{0}\right.$ and $k$ are the wave vectors of the incident and diffracted X-Rays, respectively); (b) $\Delta k$ is scanned in the $y z$ plane for measurement of the [001] axis distribution. The film coordinate axes $z, x$, and $y$ are parallel to the stretching axis, normal to the film surface, and perpendicular to them, respectively.

were clamped in a metal holder under a condition of fixed length and crystallized in the paraelectric (hexagonal) phase at $140^{\circ} \mathrm{C}$ for $2 \mathrm{~h}$ with the surfaces free from any constraint other than the tensile stress along the stretching axis. Wide-angle X-Ray diffraction (WAXD) profiles were obtained in the transmission mode by using Ni-filtered $\mathrm{Cu}-K \alpha$ radiation $(\lambda=0.15418 \mathrm{~nm})$ which was generated from a rotating anode X-Ray generator (Rigaku RU-3 SH) operated at $40 \mathrm{kV}$ and $70 \mathrm{~mA}$. A schematic diagram of the experimental arrangement is shown in Figure 1. Orientation distribution of the direction perpendicular to the $(110) /(200)$ planes and that of the [001] axis in the $\mathrm{P}(\mathrm{VDF} / \mathrm{TrFE})$ films of different thickness ranging from 5 to $200 \mu \mathrm{m}$, were measured in the course of this study.

\section{RESULTS}

An X-Ray diffraction photograph of the P(VDF/TrFE) SC film is shown in Figure 2. This "end-view" pattern projected along the stretching axis indicates strong sixfolded diffraction spots, which correspond to the reciprocal lattice points of $110 / 200$ preferentially oriented to the normal of the film surface or equivalent directions, the chain axis ( $c$-axis) being parallel to the stretching direction. In the unpoled P(VDF/TrFE) SC film, the reflections from the $\{110\}$ planes and $\{200\}$ planes are superimposed, because the ratio of the orthorhombic lattice constants, $a / b$, is very close to $\sqrt{3}$.

Figure 3 shows the rocking curve $I(\chi)$ of the 001 reflection for a $20 \mu \mathrm{m}$-thick film measured at the scattering angle $2 \theta \sim 35.3^{\circ}$ by rotating the stretching axis around the normal of the film, where $\chi$ is the angle between the $c$-axis and the stretching axis in the film plane (yz-plane). This curve shows that the [001] axis is oriented very closely parallel to the stretching axis. For a given SC film the rocking curve $I(\phi)$ of the 001 reflection scanned in the plane ( $z x$-plane), which is parallel to the stretching axis and to the normal of the film, was found to be very similar to the profile $I(\chi)$, which indicates that the orientation distribution of the [001] axis is axially symmetric with respect to the stretching axis. Therefore, the degree of orientation of the $c$-axis is ex-

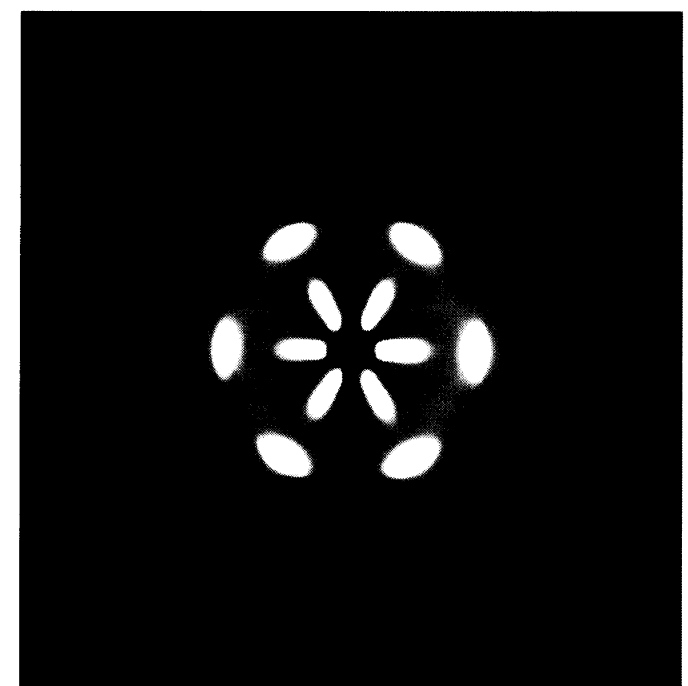

Figure 2. X-Ray diffraction pattern (end-view) of a P(VDF/TrFE) (75/25) SC film. The incident X-Ray beam is parallel to the stretching axis. The film surface is set in the vertical direction.

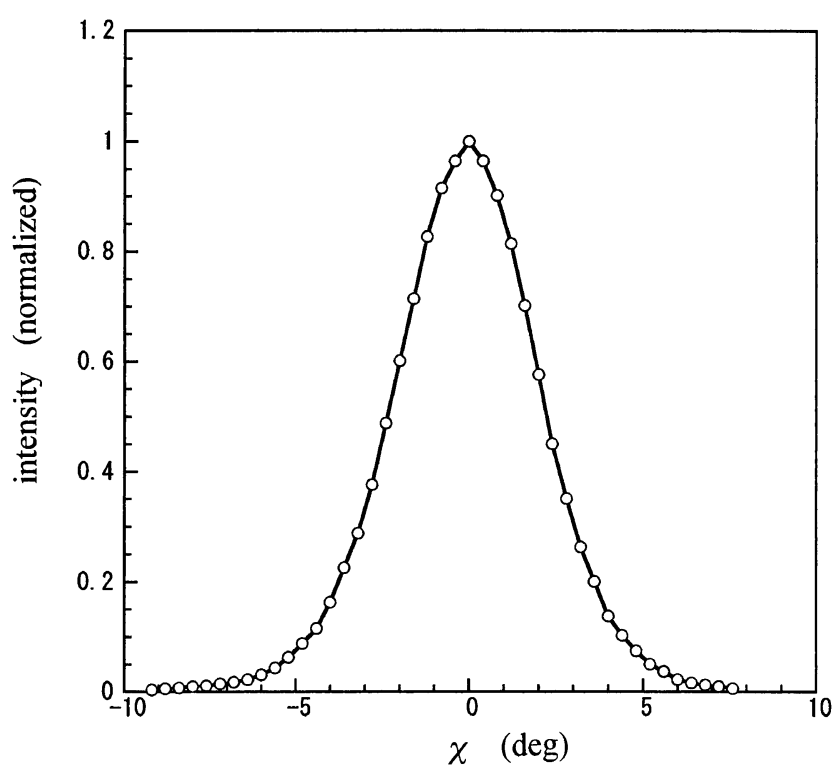

Figure 3. Rocking curve $I(\chi)$ of the 001 reflection for a $20 \mu \mathrm{m}$ thick SC film. The profile was detected by rotating the film with respect to the axis normal to the film surface at the scattering angle $2 \theta \sim 35.3^{\circ}$.

pressed by the orientation factor,

$$
f_{\mathrm{c}}=\frac{1}{2}\left(3<\cos ^{2} \chi>-1\right)
$$

where

$$
<\cos ^{2} \chi>=\frac{\int_{0}^{\pi / 2} I(\chi) \cos ^{2} \chi \sin \chi \mathrm{d} \chi}{\int_{0}^{\pi / 2} I(\chi) \sin \chi \mathrm{d} \chi} .
$$

The orientation factor $f_{\mathrm{c}}$ calculated from the observed profiles for SC films of various thickness are shown in Figure 4 . The orientation factor becomes closer to unity as film thickness decreases; the degree of orientation of 


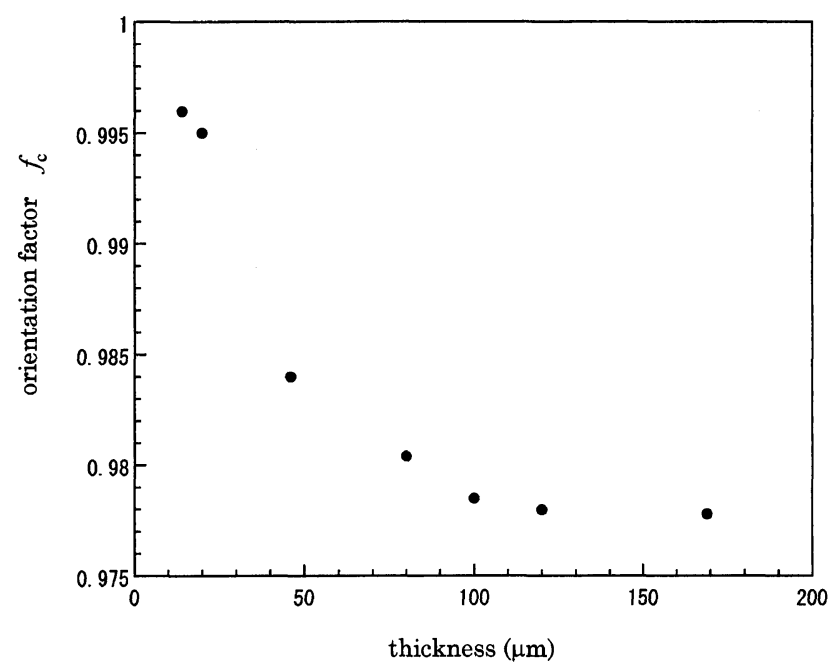

Figure 4. Thickness dependence of orientation factor $f_{\mathrm{c}}$ for the [001] axis of $\mathrm{P}(\mathrm{VDF} / \mathrm{TrFE}) \mathrm{SC}$ film. The orientation factor is calculated from the rocking curves $I(\chi)$.

the $c$-axis in the SC film is considerably higher than that in conventional crystalline polymer films.

More interesting is the fact that the axes perpendicular to the $(110) /(200)$ planes, that is, the 110/200 axes, orient very strongly to specific directions as shown in Figure 2. This is shown more clearly by the rocking curve $I\left(\omega-\omega_{0}\right)$ of the $110 / 200$ reflection in Figure 5, which was obtained from a $20 \mu \mathrm{m}$-thick SC film by rotating the film around the stretching axis with a fixed scattering angle $\left(2 \theta \sim 20^{\circ}\right)$. In Figure $5, \omega$ is the angle between the scattering vector in the equatorial plane $(x y$ plane) and the normal of the film, and $\omega_{0}=60^{\circ}$. The angular distribution of orientation is exceptionally sharp as compared to that of conventional polymer films. The width of orientation distribution becomes broader as the film thickness increases: for example, the full width at half maximum (FWHM) $\left(\Delta \omega_{1 / 2}\right)$ of the rocking profile was $13.3^{\circ}$ for a $20 \mu \mathrm{m}$-thick film, whereas $\Delta \omega_{1 / 2}=24^{\circ}$ for a $110 \mu \mathrm{m}$ film. For quantitative expression of the degree of lateral orientation distribution of the 110/200 axes with respect to the film normal or the equivalent directions, that is, the directions $\pm 60^{\circ} \mathrm{C}$ or $\pm 120^{\circ} \mathrm{C}$ from the film normal, we calculated the average value of $<\cos ^{2}\left(\omega-\omega_{0}\right)>$ from the observed rocking curves for the films of various thickness. The thickness dependence of the degree of orientation distribution for the 110/200 axes is shown in Figure 6, which indicates that the degree of lateral orientation decreases with increasing thickness of the films.

The thickness dependence of the lateral orientation of the 110/200 axes suggests that the degree of orientation of the 110/200 axes is not uniform over the whole film, but it is very high near the surface and gradually becomes lower as the distance from the surface increases. For confirmation of this suggestion, we took one SC film (180 $\mu \mathrm{m}$-thick). First we performed the WAXD measurement for the original film. We then scraped one surface of the film with a fine abrasive paper and measured the orientation distribution of the scraped film. Next, in the same way we scraped the opposite surface of this film and measured the orientation distribution. This process

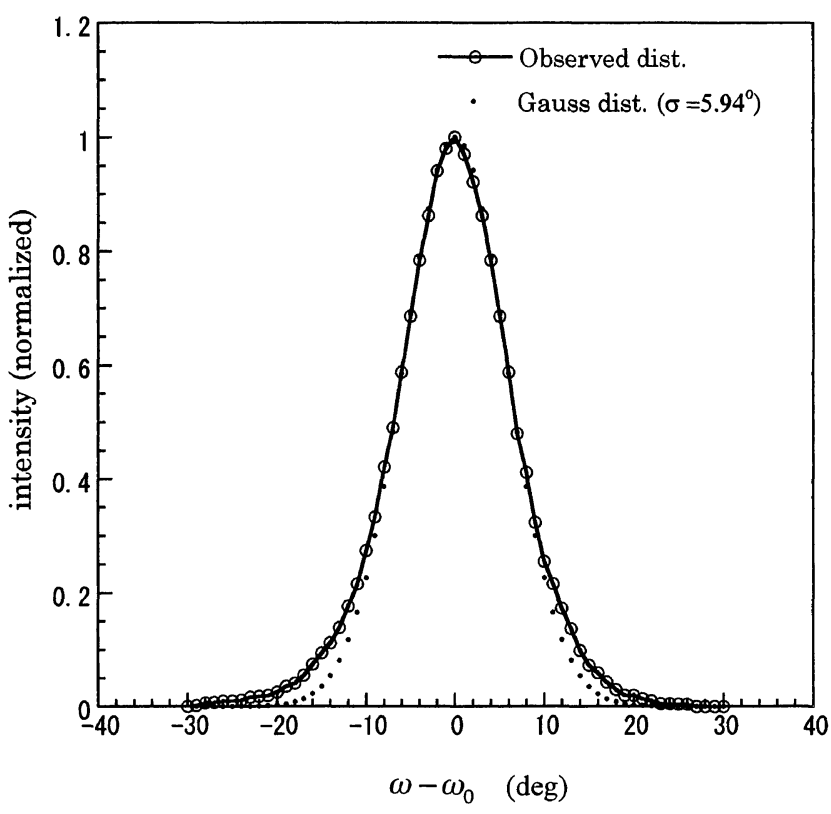

Figure 5. Rocking curve $I\left(\omega-\omega_{0}\right)$ of the $110 / 200$ reflection for a $20 \mu \mathrm{m}$-thick SC film $\left(\omega_{0}=60^{\circ}\right)$. The curve was detected by rotating the film around the stretching axis at a fixed scattering angle $2 \theta \sim$ $20^{\circ}$. A Gaussian distribution curve $\left(I=\exp \left[-\left(\omega-\omega_{0}\right)^{2} / 2 \sigma^{2}\right] ; \sigma=\right.$ $\left.5.94^{\circ}\right)$ is also plotted for comparison.

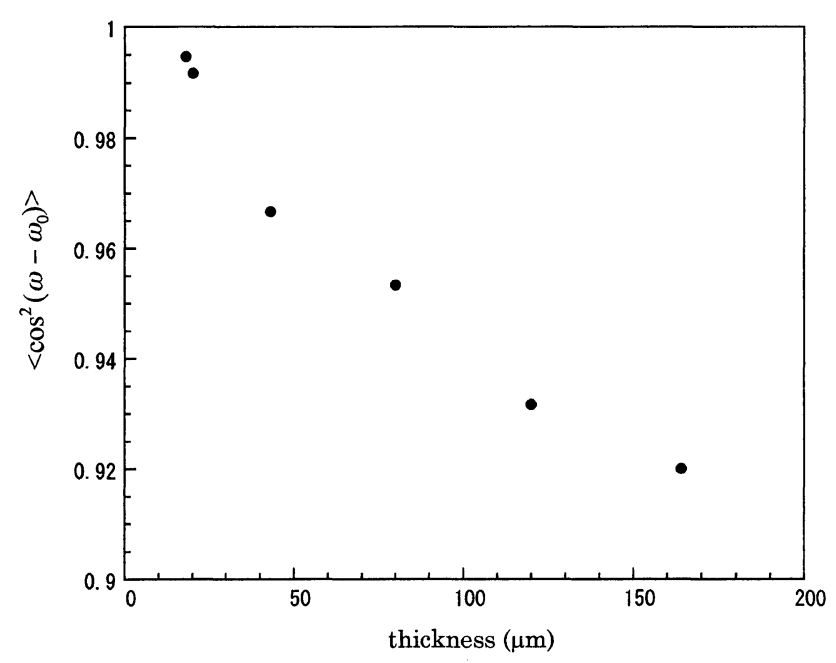

Figure 6. Thickness dependence of orientation order, $<\cos ^{2}\left(\omega-\omega_{0}\right)>$, for the $110 / 200$ axes in the plane normal to the stretching axis ( $\omega_{0}=60^{\circ}$ from the film normal). The orientation order was calculated from the observed rocking curve $I\left(\omega-\omega_{0}\right)$.

was repeated several times until the maximum possible depth was reached. The WAXD measurements were taken after every scraping of the film. The result is shown in Figure 7. The FWHM of the rocking curve increases with decreasing thickness of the scraped film. This result shows that the orientation order in the film becomes lower with increasing depth of the film.

The lateral orientational order is formed during crystallization in the hexagonal phase. Figure 8 shows the rocking profiles of the 110/200 reflection for the SC films crystallized/annealed at various temperatures. The orientational order, $\left\langle\cos ^{2}\left(\omega-\omega_{0}\right)\right\rangle$, calculated from the rocking curves are plotted in Figure 9 as a function of 


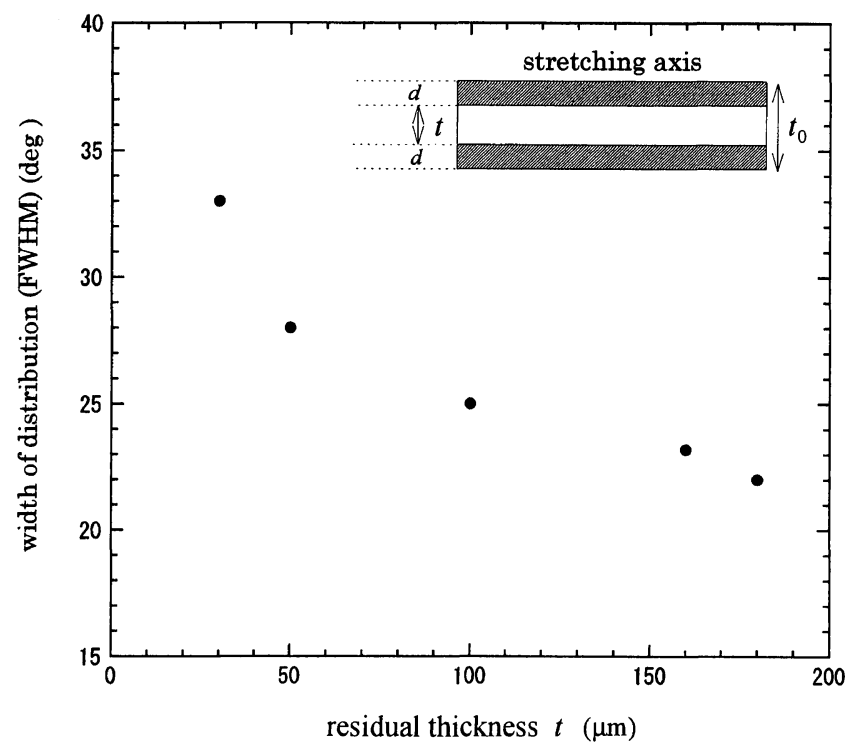

Figure 7. Dependence of orientation distribution of the 110/200 axes on the residual thickness $t\left(=t_{0}-2 d\right)$ of a scraped SC film of $\mathrm{P}(\mathrm{VDF} / \mathrm{TrFE})(75 / 25)$. The full width at half maximum (FWHM) of the rocking curve $I\left(\omega-\omega_{0}\right)$ of the scraped film is indicated as a measure of the distribution. The inset shows the geometry of the scraped film ( $t_{0}$, initial thickness; $d$, scraped thickness).

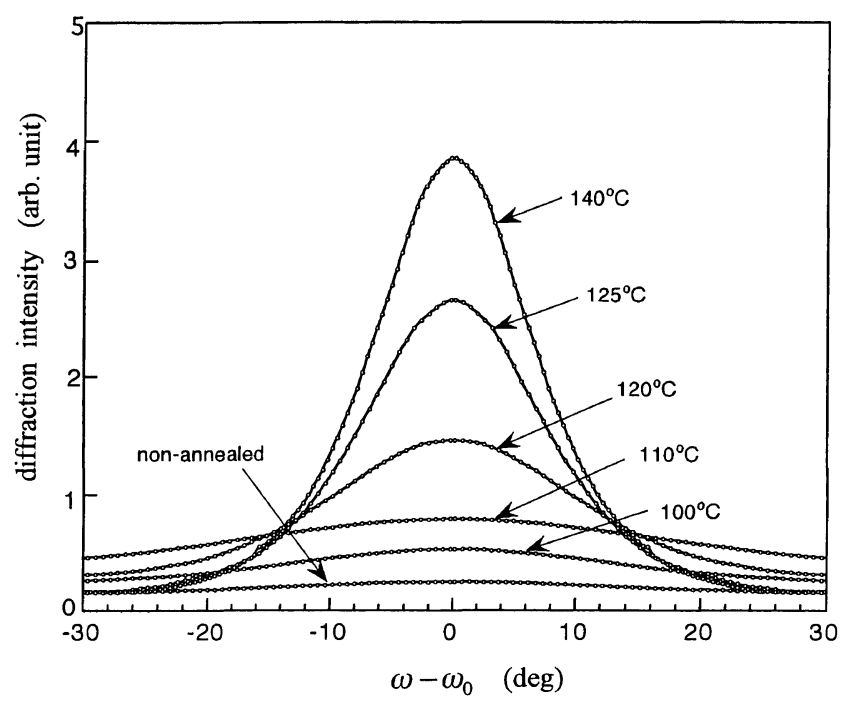

Figure 8. Rocking curves $I\left(\omega-\omega_{0}\right)$ of the $110 / 200$ reflection for uniaxially stretched $\mathrm{P}(\mathrm{VDF} / \mathrm{TrFE})$ (75/25) films crystallized/annealed at various temperatures.

crystallization/annealing temperature. It is important that growth of the orientational order is remarkable only in the films crystallized/annealed at temperatures above the Curie temperature, i.e., the orthorhombic-tohexagonal phase transition temperatrure $\left(T_{\mathrm{c}} \sim 124^{\circ} \mathrm{C}\right)$.

It was also found that the observed profiles $I\left(\omega-\omega_{0}\right)$ roughly fit to Gaussian distribution functions as shown in Figure 5.

\section{DISCUSSION}

The experimental results show that both the orientation factors for the 001 and 110/200 axes increase with decreasing thickness of the SC film, and that the orien-

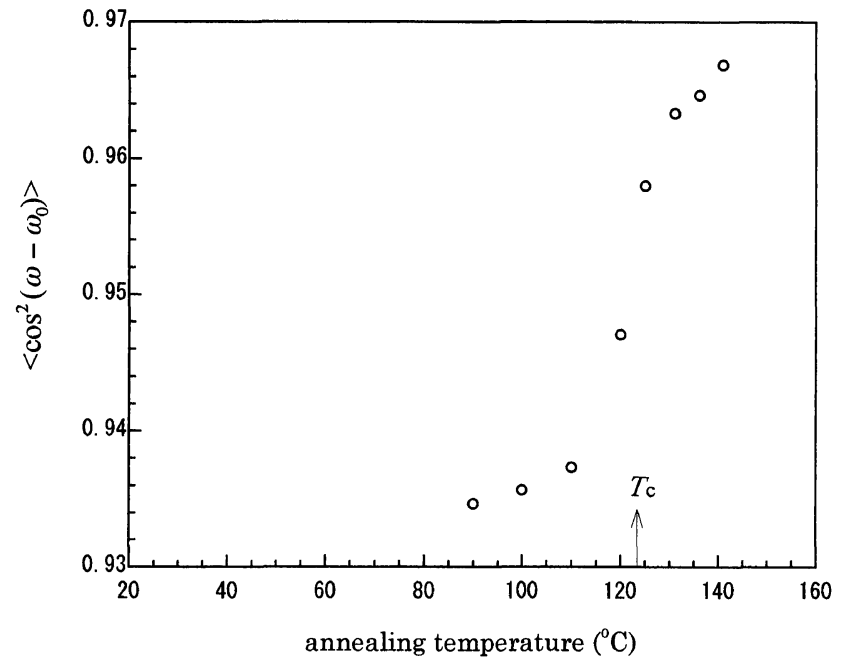

Figure 9. Dependence of lateral orientational order, $<\cos ^{2}\left(\omega-\omega_{0}\right)>\left(\omega_{0}=60^{\circ}\right)$, for the $110 / 200$ axes as a function of annealing temperature. Rapid growth of preferential orientation occurs above the Curie temperature $T_{\mathrm{c}}$.

tation order is formed in the hexagonal phase. As reported in a previous paper, ${ }^{5}$ the $\mathrm{P}(\mathrm{VDF} / \mathrm{TrFE})$ crystal in the hexagonal (paraelectric) phase is a liquid crystal: the chain molecules are very mobile along the chain axis. The chain molecules in a uniaxially stretched film diffuse easily along the stretching direction in the hexagonal phase. In the thinner film the direction of the diffusive flow of the chains in the paraelectric phase is restricted within a very limited space, which makes the chains to extend parallel to the film surface. This is most probably the reason why the thinner film has the higher orientation factor for the $c$-axis.

As to the lateral orientational order, i.e., the orientation of the 110/200 axes to the normal of the film surface or to the equivalent directions, the driving force to induce the preferential orientation is the surface energy of the hexagonal crystal, by considering that the orientational order develops in the hexagonal phase. Among the crystal surfaces the hex (100) plane, that is, the (100) plane of the hexagonal lattice, has the lowest surface energy, because this crystal plane is the plane of most closed packing of chain molecules, and the chain molecules in this plane have the smallest open area, where the chain molecules are exposed to air, as compared to the molecules in other crystallographic planes. (The surface energy will be roughly proportional to the open area.) Therefore, the preferential orientation of the hex (100) plane parallel to the film surface will be realized during annealing in the mobile phase. The hex (100) surface developed in the hexagonal phase is transformed into the orthorhombic (110) or (200) plane on cooling into the ferroelectric phase, which gives rise to the preferential orientation of the crystals, as really observed in the SC film. The arrangement of chain molecules near the film surface in the hexagonal phase is illustrated in Figure 10.

The size of each crystallite in the hexagonal phase is estimated from the X-Ray scattering to be $0.1-0.2 \mu \mathrm{m}$ in diameter in the direction perpendicular to the (100) plane. ${ }^{5}$ Due to the disorientation between the adjacent 
film surface (hex (100))

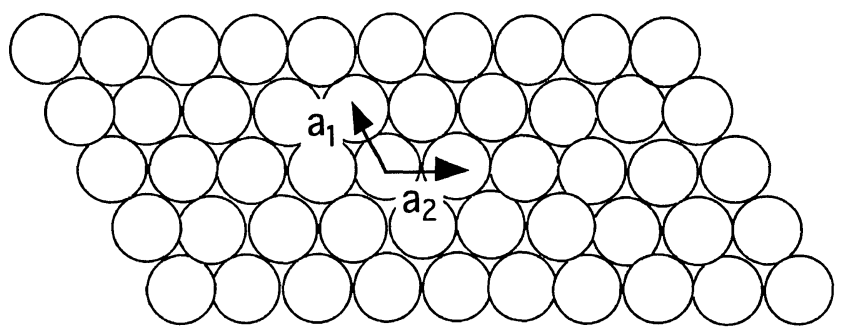

Figure 10. Molecular chain arrangements near the surface area in the hexagonal phase (projection along the $c$-axis). Each circle represents a TGTG' chain molecule undergoing rotational motion and flip-flop motion. ${ }^{1,5}$ The hexagonal (100) plane is parallel to the film surface, which is transformed to the orthorhombic (110) or (200) plane during cooling process into the ferroelectric phase.

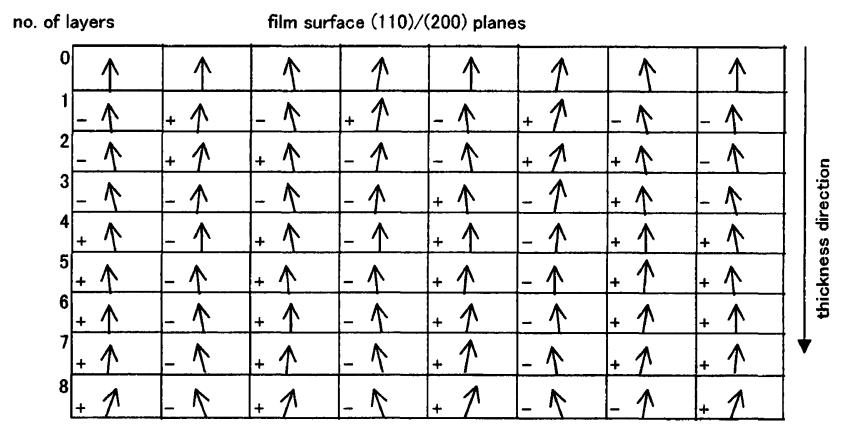

Figure 11. A proposed model for the orientation distribution of crystallites in the orthorhombic $110 / 200$ axes in a P(VDF/TrFE) SC film. Each cell and its arrow represent a crystallite and its orientation direction of the 110 or 200 axis, respectively. The sign $( \pm)$ indicates the direction (clockwise or anticlockwise) of the deviation angle $\delta$.

crystallites, the direction of the hexagonal 100 axis for inner crystallites may deviate from that of the crystallites near the film surface, which causes such orientation distribution that becomes broader with increasing distance from the film surface. When the hexagonal-toorthrohmbic phase transition occurs, the hexagonal crystallites are divided into several orthorhombic crystallites $(20-30 \mathrm{~nm}$ in diameter). Therefore, the distribution of the orthorhombic 110/200 axes becomes broader than in the hexagonal phase, as was really observed. ${ }^{6}$

With a model based on the mechanism of the chain alignment described above, we will make a simulation for the observed orientation distribution of the 110/200 axes in the SC films in the ferroelectric phase. The model, a linear orientation diffusion model, is illustrated in Figure 11. In this model, we assume that: (i) a film is divided into $2 N$ layers parallel to the film surface, each layer of which is composed of many crystallites aligning parallel to the layer, (ii) disorientation or deviation angle of the 110/200 axes between a crystallite in the $n$-th layer and the adjacent crystallite in the $(n-1)$ th layer is some small constant angle, $+\delta$ or $-\delta$; the event $+\delta$ or $-\delta$ occurs as a random process with equal probability; and (iii) the deviation of a crystallite in the $n$-th layer is independent of the neighboring crystallites in the same layer. Therefore the orientation distribution $f(\omega: n)$ of the crystallites in the $n$-th layer, where $\omega$ is the angle

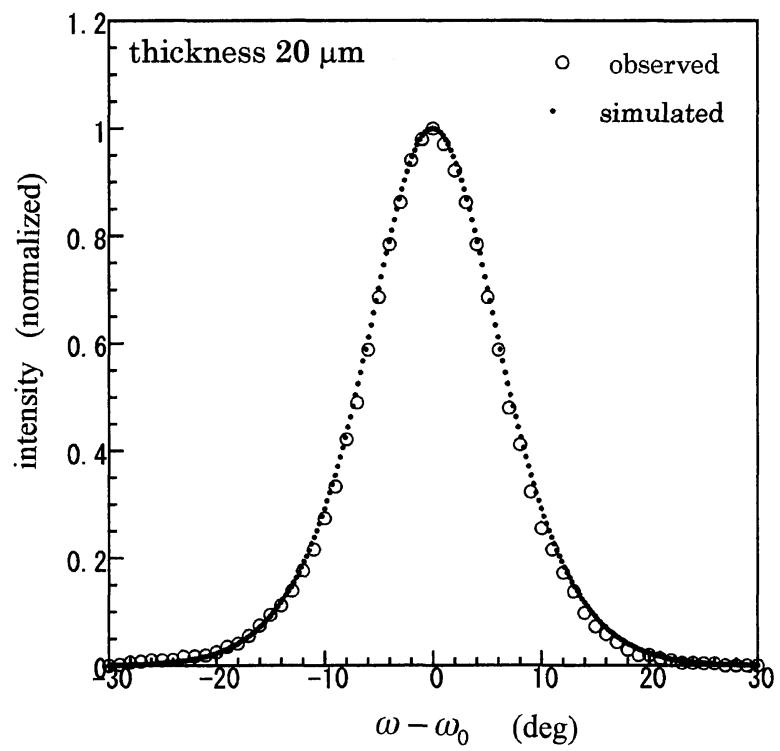

Figure 12. Fitting of simulated angular distribution of the 110/ 200 axes to the observed orientation distribution curve (rocking curve $\left.I\left(\omega-\omega_{0}\right)\right)$ for a $20 \mu \mathrm{m}$-thick film. Both curves are normalized with their maximum intensity.

between the normal of the film and the axis normal to the (110) or (200) plane of the crystallite, is expressed by a Gaussian distribution function for one-dimensional diffusion process of $n$ steps:

$$
f(\omega, n)=\frac{1}{\sqrt{2 \pi\left(n_{0}+n\right) \delta^{2}}} \exp \left(-\frac{\omega^{2}}{2\left(n_{0}+n\right) \delta^{2}}\right)
$$

In the above expression, $n_{0}$ is a number of virtual layers existing above the film surface to express the angular distribution of the crystallites in the surface layer (0-th layer). (This distribution function is the same as the endto-end distance distribution function of a onedimentional polymer chain comprising $\left(n+n_{0}\right)$ bonds of bond length $\delta$.) The observable rocking curve $I(\omega)$ is proportional to the sum of the distribution function for all the layers in the film;

$$
I(\omega)=A \sum_{n=0}^{N-1} f(\omega ; n)
$$

where $A$ is the normalization factor. Since the orientation distribution of the 110/200 axes has six-fold symmetry with respect to the normal of the film surface, $I(\omega)$ in eq 4 is also equal to $I\left(\omega \pm 60^{\circ}\right)$ and $I\left(\omega \pm 120^{\circ}\right)$.

We simulated angular distribution of crystallites to fit the observed intensity distribution (rocking curves) using eq 4 with adjustable parameters $\delta$ and $n_{0}$. In Figures 12 and 13 the simulated curves are compared with the normalized rocking curves observed for two SC films of different thickness $(20 \mu \mathrm{m}$ and $50 \mu \mathrm{m})$. In the calculation of orientation distribution of $50 \mu \mathrm{m}$-thick film, the contribution of the neighboring diffraction spots which are centered at $\omega=\omega_{0} \pm 60^{\circ}$, where $\omega_{0}$ is $0^{\circ}$ or equivalently $60^{\circ}$, was taken into account, because the thicker films have broader orientation distribution. Two simulation 


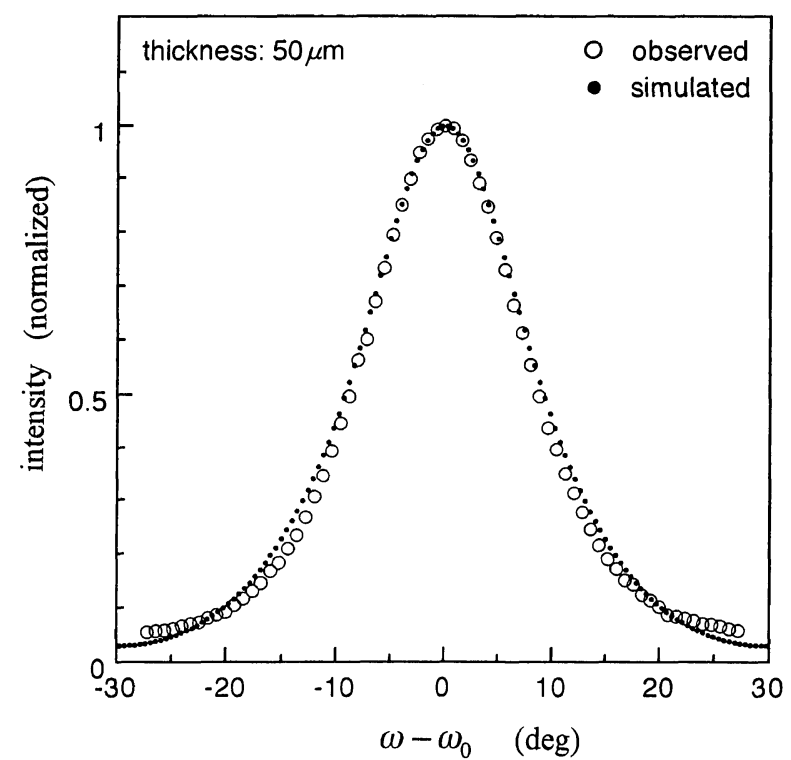

Figure 13. Fitting of simulated angular distribution of the 110 / 200 axes to the observed orientation distribution curve (rocking curve $\left.I\left(\omega-\omega_{0}\right), \omega_{0}=60^{\circ}\right)$ for a $50 \mu \mathrm{m}$-thick SC film. Both curves are normalized with their maximum intensity. The parameters used for the simulation are the same as used in Figure 12 except for $N$, which is proportional to the film thickness.

curves thus calculated using the same parameters $\delta$ and $n_{0}$ reproduce very well with the observed rocking curves for these films of different thickness; the parameters used were $n_{0}=45, N=250$, and $\delta=0.53^{\circ}$ for the $20 \mu \mathrm{m}$ film and $n_{0}=45, N=625$, and $\delta=0.53^{\circ}$ for the $50 \mu \mathrm{m}$ film. The number of $2 N$ adopted here corresponds to the layer thickness of $40 \mathrm{~nm}$. The coherent length or size of crystallite estimated from the width of X-Ray Bragg reflection of the $110 / 200$ is about $20 \mathrm{~nm}$. Therefore it seems more preferable to use $N=500$ and $N=1250$ for $20 \mu \mathrm{m}$ and $50 \mu \mathrm{m}$ films, respectively. In this case, the deflection angle $\delta$ is $0.37^{\circ}$, and $n_{0}=90$. Our recent study showed that the ratio of the orthorhombic lattice constants, $a / b$, is not exactly equal to $\sqrt{3}$, but differs by about 3\% from $\sqrt{3}$. This difference may be one of the possible causes to bring about the deviation angle between the adjacent crystallites. The validity of this deviation angle, however, must be checked in future by other techniques.

The present model is a one-dimensional model neglecting the effect of orientation of adjacent crystallites in the same layer, and therefore may be too simple to precisely analyse the preferential orientaion of the SC film. However, the present model represents fairly well the characteristics of the observed orientation distribu- tion of crystallites. Thus, we believe that the mechanism of preferential orientation is not far from the proposed model.

\section{CONCLUSIONS}

1. The $110 / 200$ axes as well as the 001 axis in the SC film of $\mathrm{P}(\mathrm{VDF} / \mathrm{TrFE})$ exhibit high orientation order. The $(110) /(200)$ planes are parallel to the film surface. The orientation order is grown in the hexagonal (paraelectric) phase, where chain molecules are mobile along the chain axis.

2. The orientation order for the 001 and $110 / 200$ axes increases with decreasing film thickness. The orientational order for the 110/200 axes is not uniform over the whole film, but decreases with increasing distance from the film surface, which suggests that the orientation in the orthorhombic phase is mainly determined by the most favorable arrangement of chain molecules at the film surface in the hexagonal phase.

3. The orientation distribution of the $110 / 200$ axes is simulated on the basis of an orientation diffusion model that the orientation of crystallites at the film surface propagates into crystallites in the inner layers of the film as a diffusion process; the orientation angle between the adjacent crystallites is deviated by a small angle $\delta$, the sign ( \pm ) of which is randomly chosen. The calculated curves for films of different thickness fit well to the observed ones.

Acknowledgments. The authors are grateful to Dr. F. Ishii of Hokkaido University for valuable discussion, and to Daikin Kogyo Co. Ltd. (Osaka, Japan) for kindly supplying the $\mathrm{P}(\mathrm{VDF} / \mathrm{TrFE})$ copolymer. This work was supported in part by Grants-in-Aid for Scientific Research (Nos. 80455448 and 10450357) from the Ministry of Education, Science, Sports, and Culture.

\section{REFERENCES}

1. H. Ohigashi, K. Omote, and T. Gomyo, Appl. Phys. Lett., 66, 3281 (1995)

2. K. Omote, H. Ohigashi, and K. Koga, J. Appl. Phys., 81, 2760 (1997).

3. J. C. Wittmann and P. Smith, Nature, 352, 414 (1991).

4. D. E. Moncton and R. Pindak, Phys. Rev. Lett., 43, 10 (1979).

5. H. Ohigashi, K. Omote, H. Abe, and K. Koga, J. Phys. Soc. Jpn., 68, 1824 (1999).

6. T. Miyazaki, M. Eng. Thesis in Materials Science and Engineering, No. 2071, Yamagata University, 1996.

7. H. Ohigashi, T. Gomyo, T. Miyazaki, and K. Koga, Polym. J., (to be submitted). 